03

\title{
Интерференция встречных волн в планарной мелкослоистой структуре „графен-диэлектрик“
}

\author{
() С.А. Афранасьев, Д.А. Евсеев, Д.И. Семенцов \\ Ульяновский государственный университет, \\ 432970 Ульяновск, Россия \\ e-mail: sementsovdi@mail.ru
}

Поступила в редакцию 08.01.2019 г.

В окончательной редакции 15.05.2019 г.

Принята к публикации 16.05.2019 г.

\begin{abstract}
Исследовано падение двух встречных когерентных волн на противоположные поверхности планарной слоисто-периодической структуры графен-диэлектрик. В длинноволновом приближении показано, что за счет изменения разности фаз падающих на структуру волн возможно управление интенсивностью уходящих волн и поглощением излучения в структуре. В терагерцевой частотной области имеются близкие к оптимальным условия для реализации эффекта модуляции интенсивности, а в ближнем ИК и видимом диапазонах возможна модуляция поглощательной способности структуры.
\end{abstract}

Ключевые слова: интерференция волн, слоисто-периодическая структура, управление интенсивностью и поглощением волн.

DOI: 10.21883/OS.2019.09.48197.4-19

\section{Введение}

В последнее десятилетие внимание исследователей привлекла идея антилазера или когерентного идеального поглотителя (КИП) (англ. - coherent perfect absorber) [1-5]. В режиме КИП слой поглощающей среды полностью поглощает энергию двух когерентных волн, падающих на его противоположные поверхности. Следует заметить, что данный эффект был предсказан еще в 1962 г. белорусским физиком А.П. Хапалюком [6]. Указанный режим является частным случаем общего интерференционного эффекта, наблюдаемого при падении встречных волн на плоскопараллельный слой (или планарную структуру) и получившего название интерференции встречных волн (ИВВ). Суть его состоит в том, что при изменении амплитудно-фазовых соотношений падающих на слой встречных волн происходит перераспределение интенсивности волн, уходящих в окружающее пространство, что делает возможным управление как интенсивностью этих волн, так и поглощаемой мощностью.

В конце 1980-х гг. были поставлены эксперименты по наблюдению ИВВ в тонких металлических пленках [7]. В последующих работах по ИВВ особое внимание уделялось прикладным вопросам модуляции интенсивности и поглощения [8-18]. С этой целью исследовались тонкие слои металлов $[17,18]$, прозрачные диэлектрики [9], a также поглощающий диэлектрик (кварц) в области сильной частотной дисперсии [10]. Интересна ИВВ в намагниченных пленках ферритов, где имеется возможность управления модуляцией с помощью внешнего магнитного поля $[11,14]$. В экспериментах последних лет по исследованию фазовых эффектов модуляции наблюдались режимы, близкие к КИП $[15,16,18]$.

В последние годы активно исследуются особенности взаимодействия с электромагнитным полем монослоев графена и слоисто-периодических структур (СПС) на его основе [19-23]. Высокая проводимость графена при практическом отсутствии поглощения в широкой частотной области, возможность локализации волнового поля в очень тонких слоях, а также управляемость внешними полями и температурой делают графенсодержащие структуры одними из наиболее перспективных материалов фотоники и оптоэлектроники.

В связи со сказанным в настоящей работе в рамках длинноволнового приближения (приближения эффективной среды) исследуются особенности ИВВ в СПС „графен-диэлектрик“. СПС представляется однородным слоем, оптические свойства которого описываются эффективной комплексной диэлектрической проницаемостью (ДП). Для режима встречных волн анализируются интерференционные эффекты модуляции интенсивности в терагерцевом диапазоне и модуляции поглощения в ближнем ИК и видимом диапазонах. В последнем случае обсуждается возможность реализации режимов, близких к КИП.

\section{Параметры структуры}

Рассмотрим плоскослоистую периодическую структуру из чередующихся слоев диэлектрика и слоев графена с толщинами $d_{d}$ и $d_{g}$. Толщина всей структуры $d=N d_{0}=N\left(d_{d}+d_{g}\right)$, где $d_{0}$ - период структуры, $N$ - число периодов. Будем считать, что длина распространяющейся в структуре волны существенно 
больше периода $d_{0}$ и может быть использовано длинноволновое приближение. Компоненты диагонального тензора эффективной ДП рассматриваемой СПС можно представить в виде $\varepsilon_{x}=\varepsilon_{y}=\varepsilon, \varepsilon_{z}=\varepsilon_{0}$, где

$$
\varepsilon=\frac{\varepsilon_{d} d_{d}+\varepsilon_{g} d_{g}}{d_{d}+d_{g}}=\frac{1}{1+\theta}\left(\varepsilon_{d}+\frac{2 i \sigma}{v d_{d}}\right), \varepsilon_{0}=\frac{\varepsilon_{d}}{1+\theta} .
$$

Здесь $\sigma$ - комплексная поверхностная проводимость графена, $\theta=d_{g} / d_{d}$ - отношение толщин слоев в периоде структуры, $\varepsilon_{d}-$ ДП диэлектрика. Величина $\varepsilon$ в (1) соответствует параллельной ориентации электрического вектора волны $\mathbf{E}$ относительно слоев структуры, а $\varepsilon_{0}-$ перпендикулярной.

Частотная зависимость проводимости графена $\sigma(v)$ в широком диапазоне частот исследована в работах [20-23]. С учетом комплексности $\sigma$ [24,25] действительную и мнимую части компоненты $\varepsilon=\varepsilon^{\prime}-i \varepsilon^{\prime \prime}$ эффективной среды представим в виде

$$
\begin{aligned}
\varepsilon^{\prime}= & \frac{1}{1+\theta}\left[\varepsilon_{d}-\frac{\sigma_{0}}{\pi v d_{d}}\left(\ln \frac{(h v+2 \mu)^{2}}{(h v-2 \mu)^{2}+\left(2 k_{\mathrm{B}} T\right)^{2}}\right.\right. \\
& \left.\left.+\frac{16 k_{\mathrm{B}} T}{h v} \ln \left(2 \cosh \frac{\mu}{2 k_{\mathrm{B} T}}\right)\right)\right], \\
\varepsilon^{\prime \prime} & =\frac{\sigma_{0}}{(1+\theta) v d_{d}}\left(1+\frac{2}{\pi} \arctan \frac{h v-2 \mu}{2 k_{\mathrm{B}} T}\right) .
\end{aligned}
$$

где $\sigma_{0}=\pi e^{2} / 2 h, e-$ заряд электрона, $h-$ постоянная Планка, $k_{\mathrm{B}}$ - постоянная Больцмана, $T$ - температура, $\mu$ - химический потенциал.

При численном моделировании процессов взаимодействия волн со структурой использовались значения $d_{g}=0.335 \mathrm{~nm}, \varepsilon_{d}=5.07$ (оксид кремния), $\theta=0.1$ и $T=300 \mathrm{~K}$. Варьировались химический потенциал $\mu$ и общая толщина структуры $d$ (т.е. число периодов $N$ ). Анализ проводился в широкой частотной области (от терагерцевого до видимого диапазона), где выполнялись условия применимости используемых моделей.

На рис. 1 представлены частотные зависимости действительной и мнимой частей $\varepsilon$, полученные при $\mu=0.2,0.4,0.6 \mathrm{eV}$ (кривые 1-3); штриховая линия отвечает ДП диэлектрика $\varepsilon_{d}$. При $v=v_{\mu}=4 \pi \mu / h$ величина $\varepsilon^{\prime}$ достигает максимального значения. В области $v<v_{\mu}$ поглощение в среде достаточно мало, тогда как на частотах $v>v_{\mu}$ СПС оказывается сильно поглощающей. С ростом частоты $\varepsilon^{\prime}$ стремится к постоянному значению $\varepsilon_{d} /(1+\theta)$. Мнимая часть $\varepsilon^{\prime \prime}$ в этой области плавно уменьшается с ростом частоты. Величина $\varepsilon^{\prime}$ меняет свой знак на частоте $v_{0}$, значение которой зависит от химического потенциала $\mu$, параметра $\theta$ и ДП диэлектрика $\varepsilon_{d}$. Область $v<v_{0}$ является областью отрицательных значений $\varepsilon^{\prime}$.

Рассмотрим нормальное падение на структуру одиночной волны линейной ТЕ-поляризации (электрический вектор волны параллелен границам раздела). Комплексные амплитудные коэффициенты отражения $r$ и прохождения $t$ вычислялись на основе известных формул

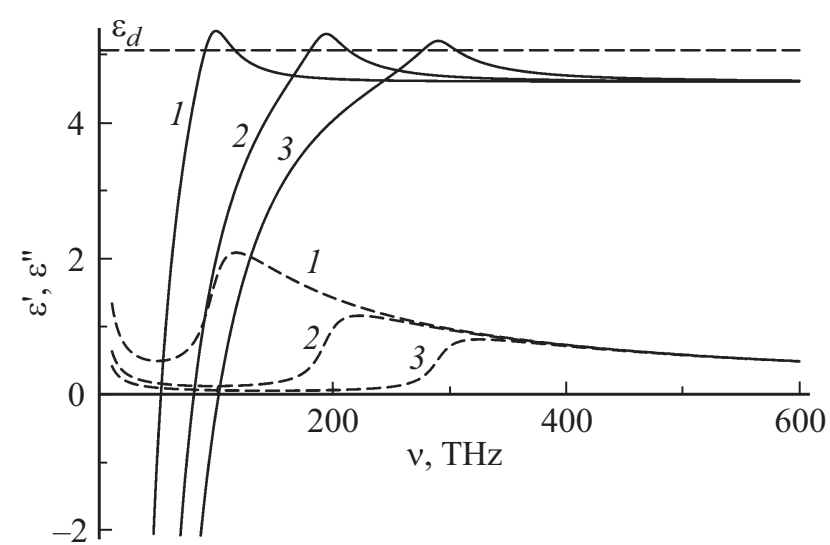

Рис. 1. Частотные зависимости вещественной и мнимой частей (сплошные и штриховые линии) эффективной ДП; $\theta=0.1, \mu=0.2,0.4,0.6 \mathrm{eV}$ (кривые $1-3$ ).

Эйри [26]:

$$
\begin{gathered}
r=i(n-1 / n) \sin \left(n k_{0} d\right) / G, t=(2 / G) \exp \left(-i k_{0} d\right), \\
G=2 \cos \left(n k_{0} d\right)-i(n+1 / n) \sin \left(n k_{0} d\right),
\end{gathered}
$$

где $n=\varepsilon^{1 / 2}-$ комплексный показатель преломления структуры, $k_{0}=2 \pi v / c-$ волновое число для вакуума, $c$ - скорость света в вакууме. На рис. 2 представлены частотные зависимости пропускательной $T=|t|^{2}$, отражательной $R=|r|^{2}$ и поглощательной $Q_{0}=1-R-T$ способностей структуры при $\mu=0.6 \mathrm{eV}$ для структур с $N=50(a)$ и $200(b)$. В соответствии с отмеченными выше особенностями частотных зависимостей эффективной ДП можно выделить три частотные области, резко отличающиеся по своим оптическим свойствам.

1. Область $v<v_{0}$ с $\varepsilon^{\prime}<0$, где волны в структуре являются сильно затухающими: мнимая часть их комплексного волнового числа $k=k^{\prime}+i k^{\prime \prime}$ преобладает над вещественной $\left(k^{\prime \prime} \gg k^{\prime}\right)$. В этой области структура является сильно отражающей и малопрозрачной при крайне незначительном поглощении.

2. Область слабого поглощения $v_{0}<v<v_{\mu}$, где структура является достаточно прозрачной для появления интерференционных максимумов и минимумов отражения и пропускания. Поглощение в этой области растет по мере приближения к ее верхней частотной границе.

3. Область сильного поглощения $v>v_{\mu}$, где для достаточно толстых структур (с числом периодов $N>100)$ поглощательная способность $Q_{0}$ превышает 0.5 и может даже приближаться к единице.

Для наблюдения эффектов ИВВ интересны частотные области 1 и 3. Область 1 с $\varepsilon^{\prime}<0$ удобна для реализации эффекта модуляции интенсивности уходящих от структуры волн. В частотной области 3 , отличающейся высокой поглощательной способностью, возможна реализация интерференционного эффекта модуляции поглощения. 


\section{Модуляция интенсивности при ИВВ}

Далее будем считать, что из вакуума на противоположные поверхности исследуемой СПС нормально падают две встречные плоские волны с одинаковой частотой $v$ и одинаковой линейной поляризацией. Падающие волны имеют амплитуды $A_{j}$, начальные фазы $\alpha_{j}$ и отвечающие им плотности потоков энергии (интенсивности) $S_{0 j}=(c / 8 \pi) A_{j}^{2}(j=1,2)$. Наряду с падающими имеются потоки энергии $S_{j}$ двух волн, уходящих от поверхностей структуры. Величины $S_{j}$ могут быть представлены в виде суммы трех слагаемых - потоков отраженной и прошедшей волн и их интерференционного потока $S_{j \text { int }}[7,8,12]$ :

$$
\begin{aligned}
S_{1} & =S_{11}+S_{12}+S_{1 \mathrm{int}} \\
& =(c / 8 \pi)\left[R A_{1}^{2}+T A_{2}^{2}+I A_{1} A_{2} \cos (\delta-\Delta)\right], \\
S_{2} & =S_{21}+S_{22}+S_{2 \mathrm{int}} \\
& =(c / 8 \pi)\left[T A_{1}^{2}+R A_{2}^{2}+I A_{1} A_{2} \cos (\delta+\Delta)\right] .
\end{aligned}
$$

Здесь $I=2(R T)^{1 / 2}-$ интерференционный параметр; $\Delta=\xi_{R}-\xi_{T}-k_{0} d-$ разность фаз, приобретаемых волнами при отражении $\left(\xi_{R}\right)$ и прохождении через слой $\left(\xi_{T}\right)$;
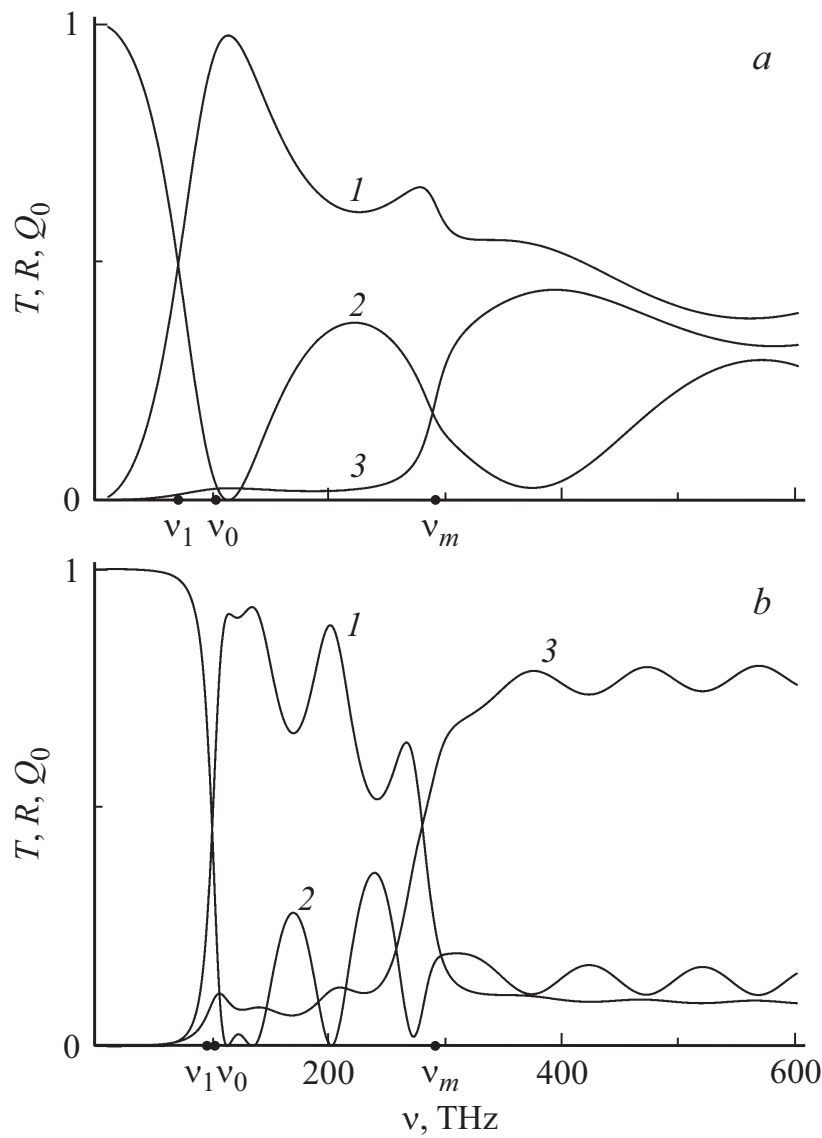

Pис. 2. Частотные зависимости пропускательной, отражательной и поглощательной способностей (кривые $1-3) ; \mu=0.6 \mathrm{eV}$, $N=50,200(a, b)$.

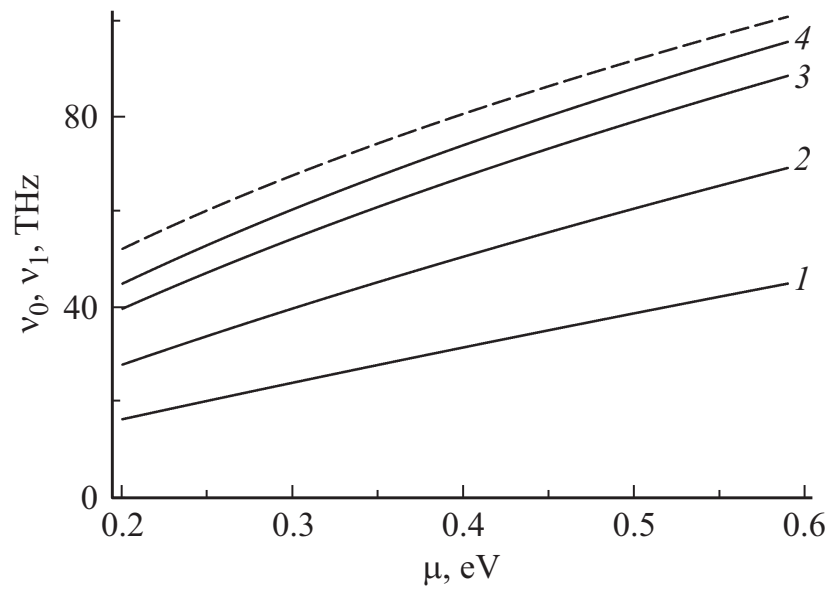

Рис. 3. Зависимости частот $v_{0}$ и $v_{1}$ (штриховая и сплошные линии) от химического потенциала графена при $N=25,50$, 100, 150 (кривые 1-4).

$\delta=\alpha_{2}-\alpha_{1}+k_{0} d-$ разность фаз волн, падающих на поверхности структуры.

Наличие интерференционных слагаемых в (4) обеспечивает возможность управления потоками $S_{j}$ уходящих от слоя волн путем изменения амплитуд и/или начальных фаз падающих волн [11,12]. Далее будем обсуждать только фазовое управление за счет изменения величины $\delta$, полагая, что $A_{1}=A_{2}=A$, так как именно в этом случае данный интерференционный эффект максимален. При вариации величин $\delta \pm \Delta$ в пределах от 0 до $2 \pi$ интенсивности $S_{j}$ изменяются от минимального значения $S_{-}$до максимального $S_{+}\left(S_{\mp}=S_{0}(R+T \mp I)\right.$, где $S_{0}$ - интенсивность каждой из падающих волн) с коэффициентом модуляции

$$
m_{S}=\frac{S_{+}-S_{-}}{S_{+}+S_{-}}=\frac{I}{R+T} .
$$

Максимальное значение интерференционного параметра $I=1-Q_{0}$ достигается для „полупрозрачного“ слоя, когда $R=T=\left(1-Q_{0}\right) / 2$. При этом условии модуляция интенсивностей $S_{j}$ происходит в пределах от $S_{-}=0$ до $S_{+}=1-Q_{0}$ с максимальным значением $m_{S}=1$. Если $\Delta= \pm \pi / 2$, то при $\delta= \pm \pi / 2$ максимум одной из интенсивностей $S_{1,2}$ совпадает с минимумом другой. Тогда для одной из падающих волн структура становится неотражающей, и вся падающая энергия (за вычетом поглощаемой части $\left.2 Q_{0}\right)$ уходит от слоя в одном направлении.

Для рассматриваемой структуры наибольшей глубины модуляции интенсивности можно добиться в области 1 $\left(v<v_{0}\right)$ с $\varepsilon^{\prime}<0$. В этой области существует единственная частота $v_{1}$, на которой $R=T$ (рис. 2). Согласно (5), это условие максимума коэффициента $I$ и соответственно коэффициента модуляции $m_{S}$. Таким образом, максимального эффекта модуляции интенсивности можно добиться подбором рабочей частоты $v=v_{1}$. Но для данной СПС того же результата можно достичь при 

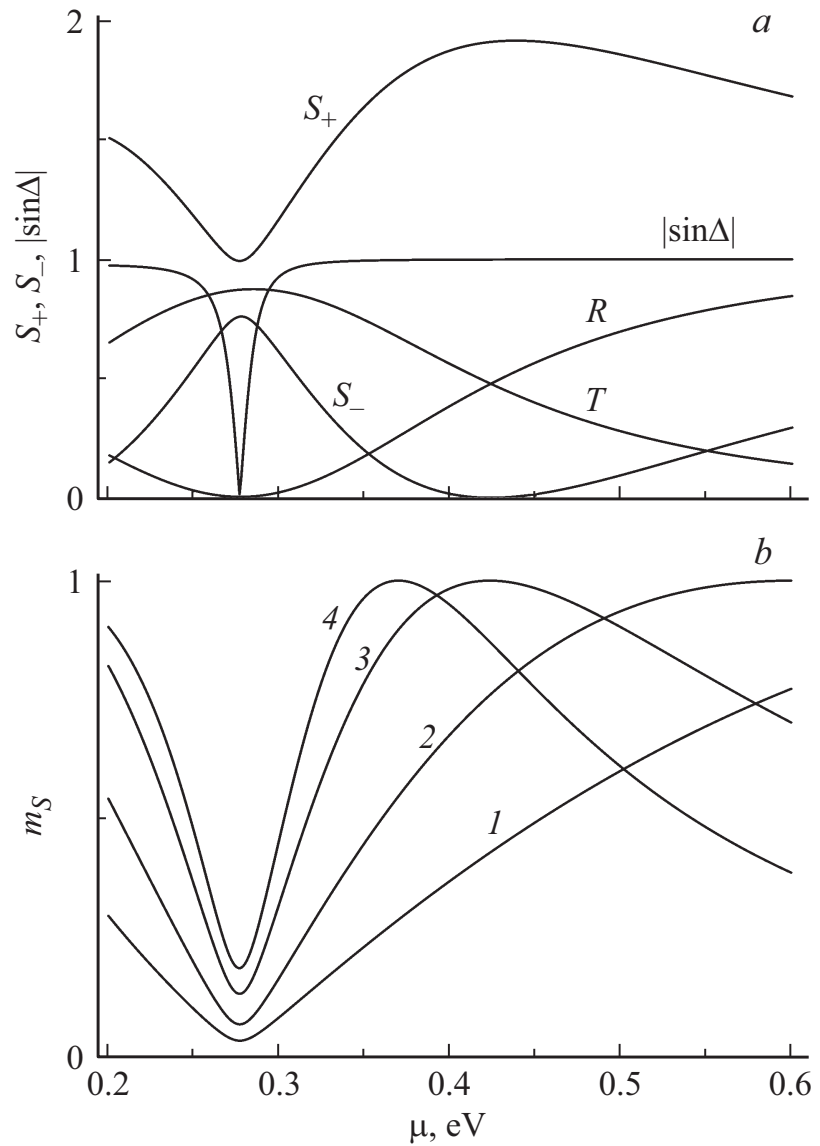

Рис. 4. Зависимости от химического потенциала графена интенсивностей $S_{+}, S_{-}$и $|\sin \Delta|(a)$, а также коэффициента модуляции $m_{S}(b) ; v=70 \mathrm{THz}, N=100(a)$ и $N=25,50,100$, $150(b$, кривые $1-4)$.

работе на фиксированной частоте падающего излучения. Изменяя внешним электрическим полем химический потенциал $\mu$ графена, можно добиться совпадения частоты $v_{1}$ с рабочей частотой.

На рис. 3 представлены зависимости частот $v_{0}$ и $v_{1}$ от химического потенциала $\mu$, полученные путем численного решения уравнений $\varepsilon^{\prime}(v)=0$ и $R(v)=T(v)$. Видно, что „оптимальная“ частота $v_{1}$ заключена в некотором частотном интервале $\Delta v$, в пределах которого она практически линейно зависит от величины $\mu$. Ширина интервала $\Delta v$ увеличивается с ростом числа периодов структуры $N$, при этом сам интервал $\Delta v$ смещается в сторону более высоких частот (зависимости 1-4 на рис. 3).

На рис. 4 представлены зависимости нормированных максимального $S_{+}$и минимального $S_{-}$значений интенсивностей $S_{1,2}$, достижимых при их фазовой модуляции, а также величины $|\sin \Delta|$ от химического потенциала $\mu$ на частоте $70 \mathrm{THz}(a)$. Нормировка $S_{+}$и $S_{-}$производится на величину $S_{0}$. Приведены также аналогичные зависимости коэффициента модуляции $m_{S}$ для четырех различных значений $N(b)$. Видно, что при $\mu=0.43 \mathrm{eV}$ выполняется условие $R=T$, когда $S_{-}=0$, а $m_{S}=1$. Зависимости рис. $4, b$ демонстрируют возможность управления величиной $m_{S}$ за счет подбора величины внешнего поля, изменяющего химический потенциал графена. С уменьшением $\mu$ рабочая частота может оказаться за пределами области $v<v_{0}$, что приводит к заметному уменьшению коэффициента модуляции.

На рис. 5 представлены зависимости интенсивностей $S_{1}$ и $S_{2}$ (в единицах $S_{0}$ ) уходящих от структуры волн от начальной фазы $\alpha_{2}$ встречной волны (начальная фаза $\alpha_{1}$ выбрана равной нулю). Расчетная частота $v=70 \mathrm{THz}$ соответствует „оптимальной“ частоте $v_{1}$ при выбранных значениях $\mu=0.6 \mathrm{eV}$ и $N=50$. Видно, что интенсивности $S_{1}$ и $S_{2}$ управляемы от нуля до максимального значения $S_{+}=1.976$ (отличие от 2 связано с наличием небольших потерь). При этом минимум интенсивности $S_{1}$ при $\alpha_{2}=0.583 \pi$ практически совпадает с максимумом $S_{2}$ при $\alpha_{2}=0.590 \pi$. Это объясняется тем, что величина $\Delta$ в частотной области $v<v_{0}$ близка к $-\pi / 2$ (кривая 3 на рис. $4, a$ ). Соответственно при $\alpha_{2}=1.583 \pi$ имеет место максимум $S_{2}$, а при $\alpha_{2}=1.590 \pi-$ минимум $S_{1}$. Следовательно, за счет подбора начальной фазы встречной волны структура может стать неотражающей для одной из падающих волн.

\section{Модуляция тепловыделения при ИВВ}

Простое перераспределение энергии падающих встречных волн в пространстве происходит только в случае $\Delta= \pm \pi / 2$. Во всех остальных случаях изменение начальной фазы хотя бы одной из падающих волн приводит к изменению поглощения падающей мощности в структуре, т.е. осуществляется его модуляция. Поглощательная способность структуры $Q$ при наличии двух падающих волн определяется как

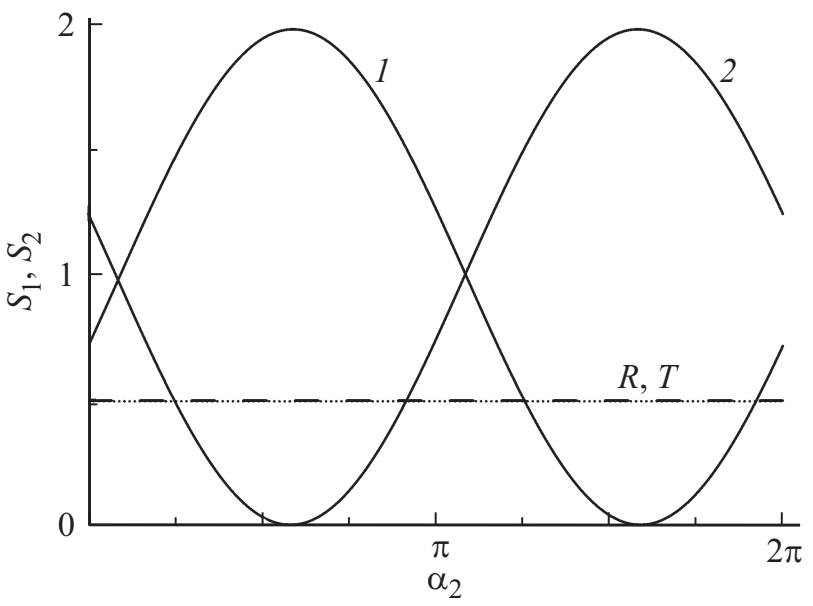

Рис. 5. Зависимости интенсивностей $S_{1}$ и $S_{2}$ (кривые 1,2 ) от начальной фазы встречной волны, $v=70 \mathrm{THz}, \mu=0.6 \mathrm{eV}$, $N=50$; штрихи - отражательная, точки - пропускательная способности структуры. 
отношение поглощаемой слоем мощности к суммарной мощности падающих волн. В оптимальном случае $A_{1}=A_{2}$ выражение для $Q$ имеет вид [12-14]

$$
Q=1-\frac{S_{1}+S_{2}}{S_{01}+S_{02}}=Q=Q_{0}-I \cos \Delta \cos \delta
$$

Интерференционные максимумы $Q_{+}$и минимумы $Q_{-}$ поглощения соответствуют значениям $\delta=0, \pi$. При этом коэффициент модуляции поглощения

$$
m_{Q}=\frac{Q_{+}-Q_{-}}{Q_{+}+Q_{-}}=\frac{I}{Q_{0}}|\cos \Delta|=\frac{I|\cos \Delta|}{1-R-T} .
$$

Подстановка в (7) максимального значения $I=1-Q_{0}$ (при $R=T$ ) дает

$$
m_{Q}=\frac{1-Q_{0}}{Q_{0}}|\cos \Delta| .
$$

В „идеальном“ случае $|\cos \Delta|=1$ поглощение может варьироваться от $Q_{-}=2 Q_{0}-1$ до $Q_{+}=1$ (полное или „идеальное“ поглощение) с глубиной модуляции $m_{Q}=\left(1-Q_{0}\right) / Q_{0}$. Таким образом, ситуация полного поглощения слоем энергии падающих встречных волн, т.е. КИП, реализуется при совместном выполнении следующих трех условий:

$$
R=T,|\cos \Delta|=1, \delta=(0, \pi),
$$

где последнее условие зависит от знака $\cos \Delta$.

Анализ эффекта модуляции тепловыделения при ИВВ будем проводить для частотной области сильного поглощения $v>v_{\mu}$ при фиксированном значении химического потенциала $\mu=0.6 \mathrm{eV}$, которому соответствует значение граничной частоты $v_{\mu}=290 \mathrm{THz}$ (длина волны $1030 \mathrm{~nm}$ ). Для частотных зависимостей оптических коэффициентов $R, T$ и $Q_{0}$ в случае падения на слой одиночной волны (рис. 2) в области $v>v_{\mu}$ характерно, что максимумы поглощения приходятся на минимумы отражения и максимумы пропускания, а „средняя“ величина поглощения $Q_{0}$ существенно зависит от толщины структуры (числа ее периодов). Поэтому имеет смысл рассмотреть эффекты ИВВ в зависимости от толщины структуры на фиксированной частоте падающего излучения.

На рис. 6 для частоты $v=375 \mathrm{THz}$ (длина волны $800 \mathrm{~nm}$ ) приведены зависимости поглощательной способности $Q$ от толщины структуры $d$ при двух значениях разности фаз $\delta=0$ и $\pi$. Этим значениям отвечают максимальная $Q_{+}$и минимальная $Q_{-}$величины поглощения, достижимые при модуляции начальной фазы одной из падающих волн (кривые 1,2). Зависимости $Q_{0}$ (кривая 3) и $R, T$, также приведенные на рис. $6, a$, напоминают соответствующие частотные зависимости на рис. 2 - они колеблются с „периодом“ $\Delta d=c / v \sqrt{\varepsilon^{\prime}}$. Той же периодичностью обладает и $|\cos \Delta|$ (кривая 1 на рис. $6, b)$ : в пределах каждого „периода“ она дважды пробегает все возможные значения от 0 до 1.

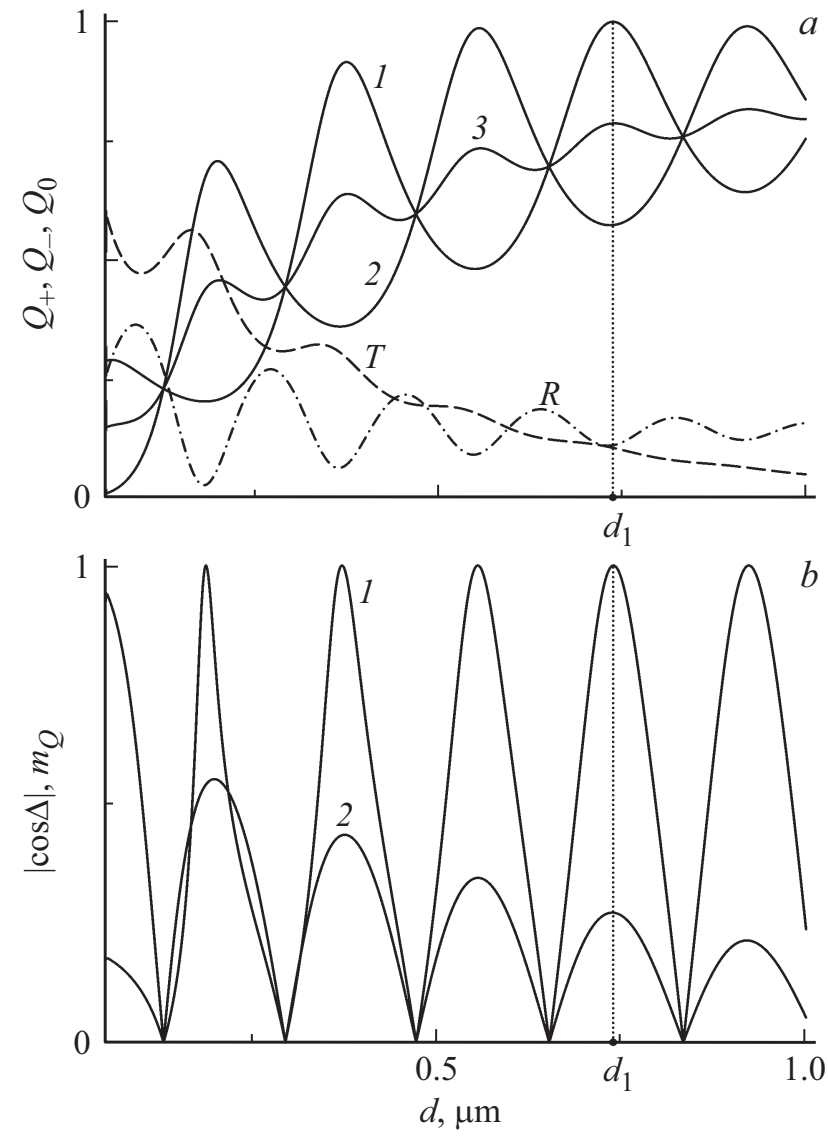

Рис. 6. Зависимости $Q_{+}, Q_{-}, Q_{0}$ (a, кривые $\left.1-3\right)$, а также $|\cos \Delta|$ и $m_{Q}(b$, кривые 1,2$)$ от толщины структуры; $v=375 \mathrm{THz}, \mu=0.6 \mathrm{eV}$.

Пики $|\cos \Delta|$ приблизительно совпадают с минимумами отражения. На эти же толщины в соответствии с (7) приходятся и максимумы $m_{Q}$ (кривая 2 на рис. $\left.6, b\right)$.

Видно, что с увеличением толщины структуры растет „Пиковое“ значение $Q_{+}$, соответствующее толщинам с $|\cos \Delta|=1$. Если на такой толщине выполняется равенство $R \simeq T$, то, согласно (9), $Q_{+}$оказывается близким к единице (режим, близкий к КИП). На рис. 6 такая толщина, равная $0.738 \mu \mathrm{m}$, обозначена $d_{1}$, ей соответствует значение $Q_{+}=0.99993$. Для пиков на толщинах как меньших, так и больших $d_{1} Q_{+}$заметно отличается от единицы.

Заметим также, что пиковый коэффициент модуляции $m_{Q}$ плавно уменышается с ростом толщины. Наибольшие значения $m_{Q}$ соответствуют небольшим толщинам, для которых, однако, поглощение мало. Как показывает анализ, увеличить поглощение для „тонких“ структур с сохранением больших значений $m_{Q}$ можно, уменьшая значение ДП $\varepsilon_{d}$, а также используя диэлектрик с достаточно большими потерями. Того же эффекта можно добиться, увеличивая долю графена в структуре, т. е. увеличивая параметр $\theta$.

На рис. 7 представлены зависимости поглощательной способности $Q$ от начальной фазы $\alpha_{2}$ встречной волны 


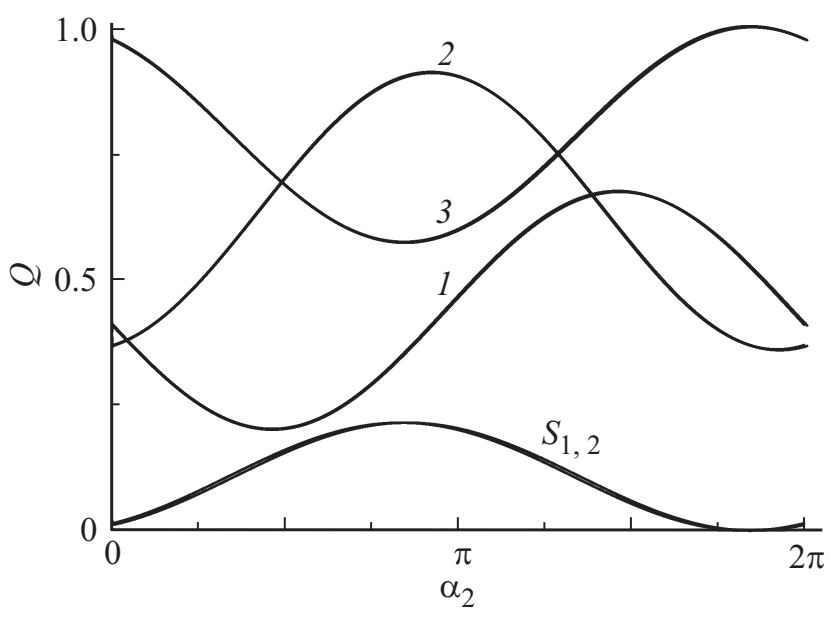

Pис. 7. Зависимость поглощательной способности $Q$ от начальной фазы встречной волны при $N=50,100,200$ (кривые $1-3) ; v=375 \mathrm{THz}, \mu=0.6 \mathrm{eV}$.

(при $\alpha_{1}=0$ ) на частоте $375 \mathrm{THz}$. Выбранные значения $N=50,100$ и 200 (кривые $1-3$ ) соответствуют первому, второму и четвертому максимумам $|\cos \Delta|$ на рис. 6 . Здесь же приведены зависимости интенсивностей $S_{1,2}$ (для $N=200$ ), нормированных на суммарную интенсивность двух падающих волн, т.е. на величину $2 S_{0}$. При точном выполнении условия $|\cos \Delta|=1$ интенсивности уходящих волн остаются одинаковыми при любой разности фаз падающих волн (для выбранных параметров это условие выполнено приближенно ввиду дискретности $N)$.

Для структуры с $N=50$ (кривая 1) поглощательная способность изменяется около среднего значения $Q_{0}=0.44$ в пределах от 0.20 до $0.67\left(m_{Q}=0.54\right)$. Для кривой 2 пределы изменения поглощения составляют от 0.36 до $0.91\left(Q_{0}=0.64, m_{Q}=0.43\right)$. В условиях кривой 3 поглощение модулируется с коэффициентом $m_{Q}=0.27$ около среднего значения $Q_{0}=0.79$ в пределах от 0.57 до практически единицы: при $\alpha_{2} \approx 1.84 \pi$ достигается максимальное для данной структуры поглощение $Q=0.9998$.

В заключение раздела отметим, что режимам, близким к режиму КИП, соответствуют невысокие глубины модуляции. Согласно (8) и (9), режим КИП с коэффициентом модуляции $m_{Q}=1$ достигается при $Q_{0}=0.5$ $(R=T=0.25)$. Но для „толстых“ структур равенство $R=T$ имеет место при значениях, меньших 0.25 . Для „тонких“ же структур, как показывает анализ, условие $R=T=0.25$ невозможно согласовать с выполнением условия $|\cos \Delta|=1$.

\section{Заключение}

В работе показана возможность реализации эффектов ИВВ в плоскослоистых структурах графен-диэлектрик. На частотах порядка десятков терагерц, соответствую- щих области отрицательных значений эффективной ДП структуры, в режиме встречных волн возможно эффективное фазовое управление интенсивностью уходящих от структуры волн. Глубиной модуляции интенсивности этих волн можно управлять двумя способами: внешним электрическим полем, изменяющим химический потенциал графена, или же подстройкой частоты падающих когерентных волн.

В ближнем ИК и видимом диапазонах структуры с достаточно большим числом слоев имеют высокую поглощающую способность $\left(Q_{0}>0.5\right)$. За счет подачи встречной волны с соответствующей начальной фазой эта величина может быть повышена до значений, близких к единице. Однако на оптических частотах затруднительно управление оптическими свойствами структуры с помощью внешнего поля. Поэтому при работе на фиксированной частоте для достижения предельно высокого поглощения необходим точный подбор толщины структуры (числа ее периодов). Если же параметры структуры заданы жестко, требуется перестройка частоты. Также отметим, что фазовый эффект модуляции поглощения для рассмотренных структур характеризуется невысокой глубиной модуляции.

\section{Финансирование работы}

Работа выполнена при поддержке Российского научного фонда и Министерства образования и науки РФ в рамках проектов № 17-72-10135 и № 3.6825/БЧ от 2017 г.

\section{Список литературы}

[1] Chong Y.D., Ge L., Cao H., Stone A.D. // Phys. Rev. Lett. 2010. V. 105. N 5. P. 053901.

[2] Longhi S. // Phys. Rev. A. 2010. V. 82. N 3. P. 031801.

[3] Pu M., Feng Q., Wang M., Hu C., Huang C., Ma X., Zhao Z., Wang C., Luo X. // Opt. Express. 2012. V. 20. N 3. P. 2246.

[4] Dutta-Gupta Sh., Martin O.J.F., Dutta-Gupta S., Agarwal G.S. // Opt. Express. 2012. V. 20. N 2. P. 1330.

[5] Розанов Н.Н. // УФН. 2017. Т. 187. № 8. С. 879.

[6] Хапалюк А.П. // Докл. АН БССР. 1962. Т. 6. С. 301.

[7] Сидоренков В.В., Толмачев В.В. // Письма в ЖТФ. 1989. T. 15. № 21. C. 34; 1990. T. 16. № 3. C. 20.

[8] Sementsov D.I., Efimov V.V. // Phys. D.: Appl. Phys. 1995. V. 28. N 6. P. 1225.

[9] Афанасьев С.А., Ефимов В.В., Семенцов Д.И. // Опт. и спектр. 1994. Т. 76. № 3. С. 475.

[10] Ефимов В.В., Семенцов Д.И. // ЖТФ. 1995. Т. 65. № 10. C. 184.

[11] Абанасьев С. А., Семенцов Д.И. // Радиотехн. и электрон. 1996. T. 41. № 12. C. 1462.

[12] Афанасьев С.А., Семенцов Д.И.// УФН. 2008. Т. 178. № 4. C. 377.

[13] Абрамов А.С., Абанасьев С.А., Елисеева С.В., Семенцов Д.И. // ЖТФ. 2013. Т. 83. № 5. С. 10.

[14] Абрамов А.С., Абанасьев С.А., Семенцов Д.И. // ЖТФ. 2014. T. 84. № 10. C. 87. 
[15] Wan W., Chong Y., Ge L., Noh H., Stone A.D., Cao H. // Science. 2011. V. 331. N 7. P. 889.

[16] Zhang J., MacDonald K.F., Zheludev N.I. // Light: Science \& Appl. 2012. V. 1. N 7. P. 18.

[17] Abramov A.S., Afanas'ev S.A., Sementsov D.I. // Eur. Phys. J. Appl. Phys. 2015. V. 72. N 2. P. 20501.

[18] Li S., Luo J., Anwar S., Li S., Lu W., Hang Z. H., Lai Y., Hou B., Shen M., Wang C. // Phys. Rev. B. 2015. V. 91. N 22. P. 220301.

[19] Castro Neto A.H., Guinea F., Peres N.M.R., Novoselov K.S., Geim A.K. // Rev. Mod. Phys. 2009. V. 81. N 1. P. 109.

[20] Фальковский Л.А. // УФН. 2012. Т. 182. № 11. С. 1223.

[21] Svintsov D., Vyurkov V., Ryzhii V., Otsuji T. // J. Appl. Phys. 2013. V. 113. N 5. P. 05370.

[22] Madani A., Entezar S.R. // Phys. B. 2013. V. 431. P. 1.

[23] Smirnova D., Buslaev P., Iorsh I., Shadrivov I.V., Belov P.A., Kivshar Yu.S. // Phys. Rev. B. 2014. V. 89. N 24. P. 245414.

[24] Evseev D.A., Eliseeva S.V., Sementsov D.I. // Eur. Phys. J. Appl. Phys. 2017. V. 80. P. 10501.

[25] Евсеев Д.А., Семениов Д.И. // Опт. и спектр. 2018. Т. 124. № 2. C. 235.

[26] Борн М., Вольф Э. Основы оптики. М.: Наука, 1973. 